\title{
Innovación tecnológica y social en turismo
}

Estudio de caso con un grupo de couchsurfers rioplatenses

\author{
Sierra, Mayra 1,3; Bacigalupe, María de los Angeles 2,3 \\ ${ }^{1}$ Departamento de Turismo, Facultad de Ciencias Económicas, Universidad Nacional de La \\ Plata, Argentina \\ ${ }^{2}$ CONICET, División Etnografía, Museo de La Plata, Facultad de Ciencias Naturales y Museo, \\ Universidad Nacional de La Plata, Argentina \\ ${ }^{3}$ mayra.sierra@econo.unlp.edu.ar, mariabacigalupe@conicet.gov.ar
}

Resumen. El presente artículo analiza la plataforma de intercambio de hospitalidad Couchsurfing como una innovación tecnológica y social tendiente a satisfacer las necesidades de los usuarios, alternativa al turismo convencional, una de cuyas características distintivas es la prestación -sin intercambio monetario- de alojamiento e integración en la vida cotidiana entre viajero/huésped/turista y residente local/hospedador. Se realiza un análisis de los conceptos de innovación, innovación tecnológica e innovación social para su posterior discusión en el ámbito del turismo. Se presentan resultados empíricos referidos al tema Innovación como motivación, con los subtemas Búsqueda de autenticidad y Turismo e Interacciones sociales, obtenidos mediante un enfoque metodológico intensivo e interpretativo y un método mixto de investigación, con predominio cualitativo. Los participantes fueron miembros de la comunidad Couchsurfing de la ciudad de La Plata con perfiles activos. Se seleccionó una muestra teórica para aplicar entrevistas en profundidad y una muestra probabilística para el completamiento de cuestionarios online y análisis documental de perfiles de usuarios de la plataforma residentes en la ciudad de La Plata. Los resultados muestran la existencia de necesidades de interacción social e intercambio cultural que motivan a las personas a participar de Couchsurfing en busca de experiencias más auténticas, que, 
según los usuarios participantes, se alcanzan a partir de sumergirse en la vida cotidiana de los locales de los lugares que visitan. La búsqueda de autenticidad constituye un factor clave y los participantes la relacionan con su distinción con el turismo convencional. Se discuten los resultados en el contexto de la idea de la plataforma Couchsurfing como turística e innovadora. Las nuevas modalidades de turismo mediadas por las tecnologías informáticas, como Couchsurfing, representan innovaciones surgidas frente a necesidades de usuarios dentro de contextos cambiantes y dinámicos y pueden considerarse formas originales de realizar una actividad humana como el turismo que, con el tiempo, también ha adquirido nuevos modos de conceptualizarse.

Palabras claves: Innovación tecnológica; Couchsurfing; Turismo; Autenticidad Recibido: 13/10/2019 Aceptado: 17/02/2020

DOI: https://doi.org/10.24215/26838559e010

\section{Technological and social innovation in Tourism}

A case study with couchsurfers in La Plata City (Buenos Aires Province, Argentina)

Abstract. In this article we study the online hospitality exchange platform called Couchsurfing as a technological and social innovation which was developed in response to a social necessity of an alternative form of tourism, different from the mass and traditional tourism. The system consists on offering housing and integration in the diary activities without monetary interchange between residents/hosts and tourists/hosted. We analyse the concepts of innovation and social and technological innovation to discuss them a posteriori in the ambit of the tourism activity. We present empirical results related to the theme Innovation as motivation and the subthemes Looking for authenticity, and Tourism and social interactions, obtained from our mixed methods research (qualitative dominant) within an intensive and interpretative framework. The participants were active 
Innovación y Desarrollo Tecnológico y Social (2020) 2 (1): 1-34

members of the Cochsurfing community which live in La Plata City, according to their profiles. We select a theorical sample to apply in-depth interviews and a probabilistic sample to complete online questionnaires and documental analysis of members' profiles. Results show the existence of necessities of social interaction and cultural exchange which are motivations for couchsurfers to participate in the community in order to get more authentic experiences that, according to participants, can be reached by the immersion in local people's lives. The look for authenticity is a key factor and makes them different from traditional tourists. We discuss the results in the context of the idea of Couchsurfing as a technological and social innovation in tourism. The new modalities of tourism mediated by informatics, like Couchsurfing, represent innovations which were born as a result of people's necessities in the changing and dynamic environments and can be considered original ways to develop a human activity like tourism which, as time passes, has acquired a new conceptualization as well.

Keywords: Technological innovation; Couchsurfing; Tourism; Authenticity

\section{Novedad u originalidad local en el conocimiento:}

Los contextos cambiantes en los que se desarrollan las actividades humanas como el turismo requieren de innovaciones que respondan a las necesidades de los sujetos. Couchsurfing es una plataforma abierta y gratuita para los participantes que surge como innovación que utiliza las tecnologías de la información y comunicación para atender estas necesidades, desarrollada por los mismos usuarios del servicio. El artículo muestra la mirada de un grupo de couchsurfers rioplatenses en sus experiencias de participar como hospedadores y hospedados a través de Couchsurfing en distintos espacios del mundo. En el desarrollo se muestra la concepción de los couchsurfers donde se diferencian de lo tradicional y convencional en el ámbito del turismo, marcando la originalidad de la propuesta. 


\section{Grado de relevancia:}

La relevancia de este trabajo radica en el análisis de un caso específico donde las tecnologías de la información y comunicación se disponen al servicio de una actividad humana como el turismo en un contexto de valoración del intercambio cultural, proponiendo a Couchsurfing como ejemplo de innovación tecnológica y social que se vincula a objetivos de algunos sectores de la sociedad relacionados con el conocimiento de otras sociedades y culturas y la búsqueda de la autenticidad en los viajes de turismo.

\section{Grado de pertinencia:}

El mantenimiento de la plataforma Couchsurfing (https://www.couchsurfing.com/) a lo largo del tiempo y la participación incrementada y que adquiere sus propias reglas de juego señala puntos favorables para considerarla como innovación con capacidad para resolver el problema de los contextos cambiantes y la negación a ciertas formas de turismo, convencionales, de masas y resistidas por algunos sectores de la sociedad (no solo por viajeros sino también por locales), atendiendo a motivaciones de personas que desean conocer otras culturas y sociedades mediante otras formas de turismo.

\section{Grado de demanda:}

Cocushsurfing comenzó en 2004 como un pequeño proyecto de sus fundadores Casey Fenton, Daniel Hoffer, Sebastian Le Tuan y Leonardo Bassani da Silveira y actualmente cuenta con catorce millones de usuarios en más de doscientas mil ciudades del mundo (cfr. https://www.couchsurfing.com/about/about-us/). 
Innovación y Desarrollo Tecnológico y Social (2020) 2 (1): 1-34

\section{Desarrollo del producto}

\section{Introducción}

Los cambios sociales actuales generan nuevos escenarios para el desarrollo de las actividades humanas que si bien tienen larga trayectoria a lo largo de su existencia se encuentran con desafíos teóricos, metodológicos y prácticos.

Una de las actividades humanas que ocupa un lugar privilegiado actualmente y que tiene sus raíces en la Revolución Industrial y los procesos de urbanización, industrialización y desarrollos tecnológicos de principios del siglo XIX es el turismo. Los cambios sociales han llevado a revisar las tipologías turísticas frente a la emergencia de nuevas formas de turismo y replantear las categorías teóricas de la disciplina, asumiendo que el turismo pone el acento en la experiencia del individuo y su rol en el sector turístico (Dujmović y Vitasović, 2015). Estos cambios han llevado a poner énfasis en nuevos valores como la autenticidad (Drumond, 2013; Dujmović y Vitasović, 2015; Gómez y Patiño, Medina y Poyuelo Arilla, 2016), hiper-realidad, lo efímero y la sustentabilidad (hyperreality, ephemerality, sustainability, cfr. Gómez y Patiño, Medina y Poyuelo Arilla, 2016), entre otros.

Dentro de estos nuevos enfoques y modalidades de turismo se halla Couchsurfing, una plataforma online sin fines de lucro que alberga una comunidad global que tiene por finalidad el intercambio de servicios de hospitalidad en sus propias residencias. Las personas que participan de la red ofrecen sus hogares para hospedar visitantes e incluso ser guías de sus propias ciudades. Aparece así el concepto de hospitalidad como la relación entre visitantes y anfitriones (Ascanio, 2007).

El presente artículo analiza a Couchsurfing como una alternativa al turismo convencional y como una innovación tecnológica y social tendiente a satisfacer las necesidades sociales de los usuarios, desde la perspectiva de los miembros de la comunidad que se identifican a sí mismos como pertenecientes a La Plata (por lo 
Innovación y Desarrollo Tecnológico y Social (2020) 2 (1): 1-34

cual hemos decidido identificarlos como miembros rioplatenses, aun cuando sus experiencias se refieren a cualquier parte del mundo).

\section{Concepto de Innovación}

El concepto de innovación puede entenderse desde la mirada de distintos autores.

El Manual de Oslo de la Organización para la Cooperación y el Desarrollo (en adelante, OCDE) señala que innovación es la concepción y aplicación de cambios significativos en el producto, el proceso, el marketing o la organización de una empresa con la intención de mejorar sus resultados y/o resolver problemas concretos. Según esta definición, los cambios innovadores surgen a partir de la implementación de nuevos conocimientos científicos y técnicos, ya sean desarrollados en el interior de la organización, con participación externa o a través de servicios de consultoría o compra de tecnología (OCDE y Comunidades Europeas, 2006). Para que un producto o proceso sea innovador no sólo debe agregar novedad, sino también tener un efecto positivo en el mercado (OCDE, 1996).

Una innovación se refiere a la transformación de una idea en un producto o equipo comerciable, nuevo o mejorado, en un proceso operativo en la industria o el comercio, o en una nueva metodología para la organización social. En este sentido, la innovación tecnológica es la que comprende los nuevos productos y procesos y los cambios significativos, desde el punto de vista tecnológico, en productos y procesos (Escobar Yéndez, 2000).

También se define a la innovación a partir de su diferencia con la creatividad. Mientras que la creatividad alude a la búsqueda de soluciones o alternativas a un problema, el proceso de innovación ocurre cuando estas ideas son producidas. Sólo las personas que dan a esa creatividad un sentido práctico, que genere un valor añadido a lo ya existente, son innovadores. Este valor añadido puede ser económico, social o de proceso (Jiménez, 2015). 
Innovación y Desarrollo Tecnológico y Social (2020) 2 (1): 1-34

No se trata solo de ser creativo: para que una idea sea considerada una innovación tiene que ser nueva y crear valor (Gibson, 2014). La innovación es fundamental para diferenciarse de la competencia y requiere desafiar lo establecido (tanto en conceptos como en métodos y procedimientos), aprovechar las discontinuidades en las tendencias, explotar los recursos dentro y fuera de la organización y comprender las necesidades de los usuarios en función de desarrollar soluciones.

Lo dicho indica que la innovación resulta importante para el éxito de un emprendimiento, considerando fundamental la capacidad de innovar constantemente para continuar en el mercado. Incluso se ha señalado que la innovación es fundamental para el crecimiento tanto de la producción como de la productividad (OCDE y Comunidades Europeas, 2006).

Sin embargo, la innovación no solo tiene que ver con la producción y la productividad, sino también con una perspectiva social que puede desdibujarse cuando de éxito comercial se trata.

Hay innovaciones sociales no orientadas a los mercados comerciales sino orientadas a la sociedad, a la cultura, el arte y otros ámbitos (Echeverría, 2008).

Así como la innovación para el Manual de Oslo se mide en referencia a valores económicos y empresariales, para Echeverría (2008) la innovación social hace referencia a valores sociales, como el bienestar, la calidad de vida, la inclusión social, la solidaridad, la participación ciudadana, la calidad medioambiental, la atención sanitaria, la eficiencia de los servicios públicos y el nivel educativo de una sociedad. Si bien estos valores no pueden ser medidos en una escala métrica como los valores económicos, pueden ser medidos en escala comparativa, vinculando sus resultados a otros parámetros sociales y de desarrollo. Estas innovaciones pueden evaluarse con relación a su impacto social, observándose si, como consecuencia de la innovación, ha mejorado la calidad de vida o el bienestar de grupos de personas con relación a su estado previo. La innovación cultural también puede pensarse como 
un tipo de innovación no necesariamente económica ni comercial: no trata sobre bienes económicos sino culturales, como el incremento de la oferta cultural, nuevas infraestructuras, cultura del ocio y del entretenimiento.

Mientras las innovaciones orientadas al mercado generan riqueza (principalmente para la empresa innovadora), las innovaciones sociales generan riqueza social (cultural, artística y educativa, entre otras formas de riqueza social).

La innovación en el ámbito del turismo puede evaluarse en términos de riqueza económica y social, sin ser formas excluyentes. Las distintas modalidades del turismo y las nuevas formas y tipologías pueden combinar ambas riquezas o privilegiar una forma por sobre otra, de acuerdo a los objetivos a los que se oriente y las formas de desarrollo que los mismos usuarios le aporten.

\section{Turismo y autenticidad}

Como actividad humana, el turismo presenta distintas formas de ser entendido de acuerdo con los cambios económicos, culturales y sociales y según los puntos de vista desde donde se lo intenta comprender.

Originalmente el turismo fue considerado un concepto asociado a las vacaciones, palabra derivada de vacante aludiendo al tiempo no trabajado, es decir, al tiempo de ocio. Esta definición surge desde el aspecto legal, desde la búsqueda de beneficios para los trabajadores y las condiciones legales de acceso al tiempo libre (Hiernaux-Nicolás, 2002). A pesar de la existencia de numerosas definiciones, el turismo suele ser asociado al concepto de turismo tradicional o de masas, aquel que implica la utilización del tiempo de ocio para realizar desplazamientos voluntarios y temporales (fuera de su entorno habitual) a grandes infraestructuras de alojamiento y esparcimiento, la demanda y consumo de servicios sofisticados, donde la principal motivación es el descanso y el placer (Ibáñez y Rodríguez, 2012). 
Innovación y Desarrollo Tecnológico y Social (2020) 2 (1): 1-34

Sin embargo, el turismo es mucho más que eso y puede distinguirse de ese enfoque, y cada día aparecen nuevas y diversas prácticas turísticas asociadas a distintos segmentos de mercado.

La práctica Couchsurfing puede encuadrarse dentro de esta nueva concepción de turismo, como alternativa al turismo convencional con eje puesto en el protagonismo de los mismos usuarios del turismo, que generan su propio servicio, y en la cual los couchsurfers no solo intercambian alojamiento, sino que ofrecen hospitalidad, tienen necesidades diferentes y se encuentran motivados por la interacción social, el intercambio cultural y la búsqueda de la autenticidad.

Como sector en constante proceso de innovación, el gran desafío del turismo es lograr que las actividades no se conviertan en rutinarias y proporcionen a los turistas nuevas emociones para generar nuevas visitas, lo cual requiere innovar constantemente (Álvarez Sousa et al, 2008).

La innovación en el turismo se asocia a los cambios sociales por los que ha pasado la práctica, desde una especie de turismo relacionada con las peregrinaciones en un contexto en que las iglesias tenían mucho poder, pasando por el auge del turismo de masas a partir de la Revolución Industrial, hasta la aparición de las nuevas tendencias vinculadas a la sostenibilidad y la tecnología (Álvarez Sousa et al, 2008).

El concepto de autenticidad constituye un tema en los estudios de turismo desde que MacCannel enunciara su importancia (MacCannel, 1975, 1976 en Terziysca, 2012 y Wang, 1999).

Según la Real Academia Española, significa acreditado como cierto y verdadero por los caracteres o requisitos que en ello concurren. En el ámbito del turismo, la autenticidad de la experiencia turística ha sido muy discutida, dado que lo cierto o verdadero suele ser subjetivo. 
Innovación y Desarrollo Tecnológico y Social (2020) 2 (1): 1-34

Algunos investigadores proponen que la demanda turística transforma las prácticas culturales en bienes de consumo, dando lugar a una trivialización de las tradiciones, fiestas, rituales y expresiones culturales (citados en Fuller, 2015). De acuerdo a lo anterior, se podría decir que toda práctica turística es inauténtica. Sin embargo, otros autores, como Cohen (1988) y Fuller (2015), sostienen que los turistas son conscientes de la homogenización de la experiencia y disfrutan de la misma sin interesarse por la cultura local, sugiriendo que esa experiencia de representación de una realidad es igualmente auténtica para sus consumidores.

En su análisis original de la autenticidad, MacCannell (1973) considera que la autenticidad se alcanza a partir del acceso de los turistas a la vida cotidiana de las personas que habitan el destino visitado, y señala que los turistas demandan autenticidad, sabiendo que no pueden acceder a ella.

En este sentido, en tanto el turismo busque reproducir prácticas culturales tradicionales para su comercialización y los turistas no sean conscientes de ello, estaríamos en presencia de una escenificación de la realidad (MacCannell, 1973), pero la experiencia desprovista de autenticidad sería percibida como auténtica. Por otro lado, en tanto los turistas sean conscientes de que dichas prácticas han sido preparadas para su consumo, sabrían que no es un escenario real o que es poco auténtico, pero su interés pasaría por otro lado: que la representación sea de calidad (Fuller, 2015).

Así como la autenticidad en el turismo constituye un tema de debate, la tecnología representa un ámbito de indagación y controversia. Las nuevas tecnologías aplicadas al turismo cambian el sector y la forma de su organización, llegando a considerar que actualmente el turismo puede estar mediado por la racionalidad tecnológica, por un lado, o el abandono de la misma, por otro, a favor de la revalorización de las emociones. Una y otra perspectiva no se excluyen (Álvarez 
Sousa et al,2008), sino que confluyen en nuevas prácticas turísticas, como podría ser el caso de Couchsurfing.

Nos preguntamos entonces qué relaciones aparecen entre Couchsurfing, la innovación tecnológica y el turismo en general.

\section{Método}

El enfoque metodológico fue intensivo e interpretativo.

Se utilizó un método mixto con predominancia cualitativa, utilizando las siguientes herramientas de recolección de datos: cuestionarios online, entrevistas en profundidad y análisis documental de perfiles activos.

Delimitamos nuestro estudio a una parte de la comunidad de couchsurfers cuya cercanía permitió el contacto presencial, a los que denominamos couchsurfers rioplatenses. Cabe aclarar que esta delimitación no significa que las experiencias de Couchsurfing de estos participantes quedaron limitadas a la región rioplatense sino que ellos se identificaban con este lugar de residencia.

Las entrevistas en profundidad constituyeron el método con mayor fortaleza de la investigación y se realizaron con 6 individuos con experiencias en la comunidad Couchsurfing. El primer entrevistado fue un informante clave seleccionado a partir de referencias profesionales por sus vínculos con la comunidad y accesibilidad para la obtención de información de interés para la investigación y los restantes entrevistados fueron seleccionados mediante la técnica de bola de nieve a partir del primer entrevistado. Los criterios de inclusión fueron haber tenido experiencias de intercambio de hospedaje a través de la plataforma, ser miembro de La Plata y tener referencias de otros usuarios en su perfil de Couchsurfing.

Los cuestionarios online tuvieron el objetivo de complementar los resultados de las entrevistas en profundidad y se utilizó información de éstas para formular las 
preguntas de los cuestionarios. La muestra para los cuestionarios se seleccionó mediante el procedimiento que se detalla a continuación.

Se realizó un muestreo probabilístico sobre la base de la población delimitada por los couchsurfers que residían en la ciudad de La Plata y contaban con referencias de experiencias personales en la plataforma. Se obtuvo como resultado una muestra mínima de 73 individuos. El cuestionario fue difundido durante 30 días en dos grupos Facebook de couchsurfers de La Plata, alcanzando un total de 70 respuestas.

Finalmente se llevó a cabo el análisis documental de 110 perfiles de couchsurfers de La Plata que contaban con experiencias alojando o siendo alojados. La selección de los perfiles se realizó por orden de aparición luego de establecer el filtro "usuarios con referencias", el cual garantizó la distinción de individuos activos en la plataforma.

Las entrevistas fueron transcriptas verbatim con el consentimiento oral de los entrevistados y se utilizaron pseudónimos para preservar su identidad. Los pseudónimos utilizados fueron: Ana, Mariana, Zorba, Mateo, Eduardo y Mercedes.

Para el procesamiento de la información y construcción de datos se utilizó el programa Atlas.ti 8.0. Se aplicó el análisis temático, un método consistente en la identificación de patrones o temas relevantes en la información cualitativa que se usan para abordar los objetivos de investigación (Maguire y Delahunt, 2017). Los códigos generados a partir del procesamiento de la información dieron lugar a la construcción de temas y subtemas, entendiendo por temas a los constructos fundamentales a describir que surgen tanto de la información empírica como del marco teórico del investigador y que vinculan conceptualmente expresiones plasmadas en los textos y otras fuentes de análisis cualitativo (cfr. por ejemplo Ryan y Bernard, 2003). 
Innovación y Desarrollo Tecnológico y Social (2020) 2 (1): 1-34

En cuanto a los aspectos éticos, se siguieron las recomendaciones del Informe Belmont (National Commission for the Protection of Human Subjects of Biomedical and Behavioral Research, 1979).

\section{Resultados}

La obtención de resultados empíricos se focalizó en el tema Innovación como motivación, con los subtemas Búsqueda de autenticidad y Turismo e interacciones sociales. Los resultados se discutieron a fin de analizar la relación entre Couchsurfing, turismo e innovación.

\section{La búsqueda de experiencias alternativas}

Para los usuarios entrevistados, su participación en Couchsurfing permitió compartir la vida local cotidiana de los anfitriones que los recibían, entrando en contacto con la comunidad, interactuando, conociendo el destino desde la mirada de ellos y logrando integrarse con la cultura que los recibía. Asimismo, según sus argumentos, les permitió tener experiencias más auténticas en la medida en que vivenciaban dicho contacto con la comunidad local, utilizando el concepto de auténtico para significar aquellas experiencias altamente personalizadas y diferenciadas del turismo tradicional.

Los entrevistados manifestaron diversas formas en que a través de sus experiencias se integraban en la vida de los anfitriones locales, compartiendo su cultura y aprendiendo de sus costumbres:

(...) ir a la casa de alguien, tener ese calor de hogar... conocer a la familia, conocer a la abuela de "Tony" y comer el fetuccini que hace la nona con la receta de la nona.

(Zorba, 46 años)

(...) el viajero de couch que vuelve te cuenta experiencias, o sea no te habla de lugares, no te habla de tal foto, de tal monumento, porque aparte con la tecnología 
eso ahora lo tenemos, te habla de experiencias, de gente que conoció, de cosas raras que le pasaron, de comidas raras, de experiencias, te habla de experiencias.

(Mariana, 41 años)

La forma de experimentar la autenticidad era integrarse en el nuevo contexto y conocer lo que los locales hacían.

En el último viaje con las chicas terminamos yendo a jaibear. Las jaibas son una especie de cangrejos, y esta familia era una familia de pescadores, entonces tenían que levantar las jaibas, ir a levantar las trampas y ponerlas. Así que nos fuimos en un botecito de pescadores en el medio del Pacifico a levantar jaibas, esas cosas no existen, ¿entendés? solo las podés hacer así si tenés una experiencia con un local.

(Ana, 25 años)

La cita anterior da cuenta de esa idea de autenticidad: el hecho de realizar actividades que no todos los turistas pueden hacer o acceder a aquellos lugares que la gente local se guarda para sí mismos le otorga una sensación de genuino, propio, original y auténtico, de primera mano, no preparado sino espontáneo, a lo que se estaba haciendo.

(...) digamos que con las personas de Couchsurfing, en general vos vas, y ellos saben qué recomendarte: andá a tal mercado que no aparece ni en los mapas, pero ahí vas a comer la mejor comida de Chiloé. Listo, vas ahí, y, sí, comés la mejor comida de Chiloé. Hay gente que sabe, porque es su casa.

(Ana, 25 años)

Zorba compartió las mismas ideas que Ana y señaló que el couchsurfer se veía motivado por la posibilidad de acceder a los lugares cotidianos de la gente local, a esos lugares desprovistos de turistas:

Está fuera de lo que es el turismo, digamos ... Lo que a mí me atrajo de Couchsurfing es que la esencia no era esa, que la esencia era compartir, disfrutar, conocer 
Innovación y Desarrollo Tecnológico y Social (2020) 2 (1): 1-34

culturas, pero en serio culturas, no decir con el tour que me llevan a Sudáfrica y me bailan cuatro negros que salen de ahí, se sacan esa ropa y están con camisa o con jean y van a comer un McDonald's, sino, yo voy a ver las danzas zulús del que vive en la choza, el de en serio... que es más genuino.

(Zorba, 46 años)

Los argumentos extraídos del análisis documental también dieron cuenta del uso del término auténtico para describir las experiencias que propone Couchsurfing:

Me gusta conocer la visión local de los lugares a los que viajo, la cual me hace descubrir la versión más real de la mano de sus residentes. El intercambio cultural que ofrece Couchsurfing hace que mis experiencias sean aún más auténticas, siento que el poder sumergirme por unos días en la rutina de las personas locales me enriquece enormemente personal y culturalmente. Aprendiendo, compartiendo y viviendo momentos únicos.

(Perfil n87, análisis documental)

A mis 31 años decidí salir a andar con la bicicleta. Viajar, conocer culturas y paisajes diferentes, rodar y vivir cada momento de la forma más auténtica y real posible para contactar con anfitriones y pasar un buen rato, para hospedar viajeros y dar una mano en el camino.

(Perfil n¹06, análisis documental)

Lo genuino o auténtico estaba en la vida privada de las personas que habitaban el destino que, como sector de intimidad local, no estaba expuesto al turista.

Desde nuestro análisis, se podría revisar el concepto de autenticidad y qué es genuino o no, considerando si el calificativo genuino, como otros términos del lenguaje y la cultura, significa lo mismo a lo largo del tiempo o cambia en función de los contextos. ¿Por qué los negros bailando que se van a comer a McDonald's no son genuinos? Quizás conservan sus prácticas culturales, ritos y bailes que vienen 
Innovación y Desarrollo Tecnológico y Social (2020) 2 (1): 1-34

de los antepasados, pero también consumen prácticas occidentalizadas de sus entornos actuales y que con el tiempo incorporaron como parte de su cultura. La cultura, como algo genuino, no constituye un conjunto de ruinas y cosas materiales simplemente, sino es el hacer de las personas involucradas y en ese sentido es dinámica y se actualiza en cada nuevo acto y en cada nuevo sujeto que la práctica (para una perspectiva de la diversidad semántica del término cultura puede verse por ejemplo Spencer-Oatey, 2012).

\section{Couchsurfers: distinción de los turistas}

Además de diferenciar sus prácticas del turismo tradicional, los couchsurfers se autodefinieron como viajeros y no como turistas. El término turista fue utilizado mayormente con una connotación negativa y haciendo referencia exclusivamente al turista tradicional que planifica su viaje y compra paquetes estandarizados. Asimismo, asociaron al turista como consumidor de experiencias falsas o escenificadas y al viajero como aquel que alcanzaba el conocimiento de lo real. Esta distinción parece ser propia de los couchsurfers ya que en el ámbito de la literatura el consumo de experiencias mercantilizadas no necesariamente combate la autenticidad, ya que ésta es una sensación subjetiva (Cohen, 1988).

A juicio de los couchsurfers entrevistados, la experiencia era más auténtica cuanto más se alejaba de las actividades realizadas por los turistas.

Está fuera de lo que es el turismo, digamos... "Mira... sí venís a La Plata anda a comerte unas milanesas a lo de Edgardo en 17 y 71 . No te lo pierdas porque no aparece en ninguna guía de turismo, pero vos tenés que probar esa milanesa, porque esa es la verdadera de acá". Si vos venís como turista a la Argentina, es posible que veas un montón de parrillas y restaurantes en Puerto Madero y no vayas a comerte un choripán o una bondiolita a la costanera. Y ahí está la esencia de eso... y yo quiero comer un Frankfurt en Berlín donde van los Berlineses a comerlo. No en Alexander Platz que esta toda la parte de turismo, ¿entendés? 
En general cuando viajo siempre soy muy de escaparle a los hoteles o a las cosas muy (no sé cómo se dice) donde va todo el mundo no me gusta ir, entonces está bueno, porque conoces bien a la gente, conoces sus costumbres, las costumbres locales.

(Ana, 25 años)

Se destaca la diferenciación personal que los couchsurfers hicieron de su forma de viajar en comparación con el turismo tradicional. Todos los entrevistados, con distintos argumentos, manifestaron no considerarse turistas.

Desde el punto de vista de Zorba, Couchsurfing constituía un programa de intercambio cultural que, a su criterio, no era una práctica turística:

Es un programa de intercambio cultural internacional que está lejos de lo que es turismo, es de viajeros... ahí hay una diferencia, y que quien realmente siente el espíritu de Couchsurfing es tener amigos en todo el mundo, aunque vos todavía no los conozcas personalmente. Es una cosa más o menos así.

(Zorba, 46 años)

Podemos observar que en la cita anterior el entrevistado hizo la diferencia entre turista y viajero y sostuvo que los couchsurfers eran viajeros con amigos dispersos por el mundo. También aclaró por qué consideraba que el intercambio simbólico era más importante que el intercambio monetario:

Lo bueno del intercambio es que... a diferencia de lo monetario es que si nosotros nos encontramos "yo te doy una moneda vos me das una moneda", los dos nos vamos con una moneda, ahora si nos encontramos...y "yo te doy mi idea, y vos me das tu idea" los dos nos vamos con dos ideas. Y esa es la gran diferencia. 
Innovación y Desarrollo Tecnológico y Social (2020) 2 (1): 1-34

Del mismo modo, Matteo diferenció al viajero del turista y destacó la variedad de experiencias que un individuo podía tener como viajero, a diferencia del turista:

Hay para mí dos formas de viajar: cómo te decía "tipo turista", entonces... vos querés ir, relajarte, divertirte y por ahí conocer algo...y entonces si vos vas a Cuba, Europa, Estados Unidos... Sudeste Asiático es lo mismo... vas y querés relajarte, vas con tus amigos, con tu familia, con tu pareja, con tus hijos, solo... y vas a hacer algo de cultura, diversión y cosas así. Y otra forma que es como viajero... ahí cada viaje es único porque vas y conoces Cuba con la gente de Cuba, te quedas con ellos, hablas, entendés como viven, por qué viven así... qué es lo que está bueno y lo que está malo, lo que le gusta y lo que no le gusta... cómo ven ellos tu país y entonces cada viaje es diferente, en Cuba tenés una experiencia, en Estados Unidos tenés otra, en Europa tenés otra...y eso.

(Mateo, 34 años)

Marina y Mercedes también esgrimieron la diferenciación entre turistas y couchsurfers, estos últimos denominados como viajeros:

Una de las características de los viajeros es, creo, la tranquilidad y muchas veces el no planear. Es muy raro, hay en couch, pero es muy raro que te encuentres alguien de couch que haga un viaje donde diga bueno tal día a tal hora me voy a tal lugar que conozco y ta ta... no. El viajero va con la idea esta de que bueno, vamos a ver qué pasa.

(Marina, 40 años)

Si me parece que la gente que está en Couchsurfing tiende a ser más bien viajero, más que turista, ¿no? por esto que te digo de que busca quizá conocer el lugar o la gente de una forma por ahí diferente a lo que es el turismo tradicional.

(Mercedes, 33 años) 
Ana hace más hincapié en la diferencia entre el tipo de viaje que realizan los couchsurfers y otros más convencionales:

Couchsurfing está más adaptado a mi forma de viajar, que es la de conocer gente, y conocer gente del lugar. Si busco salir de fiesta o tener ruido todo el tiempo probablemente me vaya a un hostel. Creo que tiene esa diferencia.

(Ana, 25 años)

Esta distinción entre turista y couchsurfer también apareció en el análisis documental de perfiles de la plataforma:

Porque es una propuesta interesante la de conocer gente mientras uno viaja y está lejos de los suyos. La mejor experiencia para conocer lugares, comidas, costumbres y salir un poco del turismo comercial.

(Perfil n 68, análisis documental)

Couchsurfing es una plataforma ideal para la gente que vive los viajes de una forma distinta. Para los que distinguen entre turismo y viaje.

(Perfil n³5, análisis documental)

Para probar una nueva forma de viajar y conocer lugares, gente, costumbres.

(Perfil n 76, análisis documental)

Tal como se observa, todos los argumentos hacen énfasis en la diferenciación del turismo tradicional, que es la que, a su juicio, les permite tener experiencias más auténticas.

Además de destacar la búsqueda de experiencias alternativas, estos resultados dan cuenta de la distinción intencional que hacen los couchsurfers donde no quieren ser identificados con el turista convencional y lo que ellos denominan puestas en escena del turismo tradicional, muy diferente a la intimidad local. 
Innovación y Desarrollo Tecnológico y Social (2020) 2 (1): 1-34

Los resultados de los cuestionarios también mostraron esta distinción entre el turismo tradicional y la modalidad Couchsurfing: mientras el 91,4\% de la muestra definió a Couchsurfing como una plataforma que promovía el intercambio cultural, sólo el 1,4\% sostuvo que era otra forma de hacer turismo. Sin embargo, esta característica propia de los couchsurfers, la de distinguirse de los turistas y del turismo, puede repensarse. Es claro que lo que los couchsurfers denominan turismo se asocia al turismo tradicional y de masas, fenómenos que actualmente se ve muy resistido bajo las concepciones de turismo-fobia y otras similares (cfr. por ejemplo Milano, Novelli y Cheer, 2019 y Pérez-García y García Abad, 2018). Sin embargo, algunas características del turismo, aún definido de modo tradicional, puede observarse en sus actitudes de viaje: se trasladan fuera de su entorno de viaje, pernoctan y si bien no pagan un alojamiento convencional, consumen servicios turísticos, como visitar íconos turísticos: el 36\% de la muestra manifestó visitarlos frecuentemente en sus viajes y el $41 \%$ dijo hacerlo muy frecuentemente.

\section{Las interacciones sociales y el turismo}

Como más arriba señalamos, la búsqueda de experiencias alternativas más auténticas resultó ser una motivación a la hora de elegir la modalidad Couchsurfing para viajar y conocer otros espacios del mundo. Otra de las motivaciones que aparecieron para participar en la comunidad Couchsurfing fue la idea de conocer personas de todas partes del planeta para poder interactuar. Esta necesidad constituye parte de la búsqueda de la diferenciación y la autenticidad, ya que se supone que, al tener experiencias de interacción con personas nativas en el contexto de sus vidas cotidianas, los viajeros tendrán experiencias únicas y personalizadas, lo cual las haría reales y auténticas para los couchsurfers.

La idea del intercambio personal y la diferenciación en los couchsurfers se relacionó con la consideración de que el turismo tradicional construía la experiencia con una puesta en escena montada para mostrar, muy distinta a lo local. Esta idea se 
Innovación y Desarrollo Tecnológico y Social (2020) 2 (1): 1-34

observó en numerosos argumentos, tanto de los entrevistados como en los perfiles indagados en el análisis documental:

Me gusta conocer la cultura de raíz de los latinoamericanos, conocer desde lo más profundo, pasar momentos amenos, compartir lo cotidiano e intercambiar la cultura argentina. Compartir charlas, momentos, experiencias.

(Perfil n 109, análisis documental).

Couchsurfing gives me the possibility to meet people, to know real culture in different places, to open my mind, to enrich each other in every encounter.

(Perfil n49, análisis documental)

I'm on CouchSurfing to learn about the world through new and interesting people, to really get to know every place I go through a local's point of view. It's not the same travelling and staying in hotels where you remain isolated. As I usually travel alone getting to know people here in CS and on hostels makes it much more meaningful and richer. This is such an amazing community! I love meeting other solo female travelers, so we can learn from each others experiences!

(Perfil n 80, análisis documental)

Tenemos la curiosidad a flor de piel, en cada viaje que hacemos caminamos las ciudades hasta que nos duelan los pies, nos gusta perdernos en sus calles, conocer la música y comidas típicas, los sitios que frecuentan los locales y no sólo aquellos que visitan los turistas. Es por eso que creemos que Couchsurfing es una oportunidad para no desaprovechar y que puede enriquecernos enormemente.

(Perfil n²8, análisis documental) El viajero vive como realmente es. No busca que sólo le muestren una parte, busca que le muestren la realidad. Y es por eso que para el turista siempre todo está bien y el trayecto de felicidad siente que dura poco, porque es una fantasía. El viajero adapta un estilo de vida, el cual tiene sus altibajos como cualquier otro. 
Innovación y Desarrollo Tecnológico y Social (2020) 2 (1): 1-34

(Perfil n³5, análisis documental)

Conocer la cultura actual y sus protagonistas, la visión local, la versión más real de la mano de sus residentes, la realidad total, no sólo la parte que se muestra a los turistas, esas parecen haber sido motivaciones vinculadas con el intercambio que privilegiaron los viajeros de los perfiles analizados cuando eligieron viajar mediante la modalidad de Couchsurfing. En estos argumentos queda claro que, para los couchsurfers, el intercambio cultural se asoció a la autenticidad y a la distinción con el turista tradicional, que no veía la realidad sino la imagen que le mostraban, mientras que los couchsurfers, viajeros (como se autodenominaron), alcanzaban la experiencia más auténtica y real posible.

Si bien la plataforma Couchsurfing fue creada como un servicio de intercambio de alojamiento gratuito, han sido los propios usuarios quienes han definido el sentido del espacio, poniendo el énfasis en la necesidad colectiva de interacción social, hospitalidad y el intercambio cultural y simbólico que surge del propio uso de la plataforma.

En las entrevistas surgió el tema del sentido de Couchsurfing como espacio de intercambio y pudo saberse que los miembros que inicialmente participaban por la gratuidad del alojamiento podían seguir dos caminos, o cambiaban su actitud a una mirada más interactiva con el hospedador u hospedado, o terminaban siendo excluidos o autoexcluyéndose, construyendo una reputación negativa en la web. Esta forma de exclusión puede observarse en los siguientes argumentos:

Hay gente que por ejemplo te acepta en la casa, te dice "Sí te recibo" pero trabaja todo el día, o no está nunca, y vos decís " y no, porque en realidad si voy a la casa es para conocer la cultura de la gente, si no voy a poder compartir con ellos, más vale busco a otro".

(Ana, 25 años) 
Si quieres quedarte en mi casa, demuestra que hay un interés por conocerme, por conocer mi perfil, por conocer mis requisitos y si no lo leíste, y es un mensaje más genérico obvio que no (...).

(Eduardo, 29 años)

(...) muchos dicen "bueno si vos buscas esto, yo no soy la persona apta para esto entonces no me manden mensajes..." o muchos hacen... para evitar que uno mande el mismo mensaje a todos, dicen en su perfil "cuando me mandas una solicitud o algo tenés que poner esta palabra así sé que leíste mi perfil o cosas así".

(Mateo, 34 años)

Entonces pongo, dentro de la descripción blablá y en un momento pongo: "si me vas a pedir couch..." que me pidan con tiempo, con determinado tiempo digamos y que en la solicitud escriban una frase secreta, que creo que es Carpe Diem, muy trillado lo mío, pero bueno (risa). Eso es para asegurarme que han leído el perfil porque mucha gente hace tititi, y como te decía: manda solicitudes (...).

(Marina, 40 años)

Tal como expresaron Mateo, Marina y Eduardo, los mismos usuarios han creado un método de exclusión a través de palabras clave, esto es para asegurarse que las personas han leído los perfiles y conocen al anfitrión a quien están solicitando hospitalidad, de modo que todos aquellos que no han leído el perfil y envían un mensaje genérico de solicitud quedarán excluidos automáticamente por no haber utilizado la palabra clave.

La importancia de establecer relaciones sociales e interactuar con los anfitriones que los acogen o los huéspedes que los visitan también se manifestó en los resultados de los cuestionarios, siendo de las actividades más valoradas utilizar el transporte público y viajar con la comunidad local (64\%), pasear con gente local y que me cuenten su historia (51\%), conocer las costumbres de otros couchsurfers 
Innovación y Desarrollo Tecnológico y Social (2020) 2 (1): 1-34

(51\%), compartir una comida casera con un local (46\%) y conversar durante horas con otro couchsurfer (43\%).

Para los couchsurfers la participación en este sistema de intercambio cubría una necesidad surgida del propio uso de la plataforma: la necesidad de interactuar y conocer personas de otras partes del mundo para intercambiar su cultura y, de esta manera, tener experiencias únicas y genuinas. Su idea de autenticidad se vio vinculada a la experiencia que les otorgaba el intercambio de hospitalidad en la plataforma. La distinción realizada con los turistas, su rechazo a pertenecer e identificarse o ser identificados con ese grupo de consumidores de servicios turísticos tradicionales, fue mostrado reiteradas veces por los entrevistados y los perfiles analizados.

En este punto se puede observar una tensión con lo que ciertos autores señalan cuando se refieren a que los viajeros utilizan el turismo como búsqueda de satisfacción a su necesidad de autenticidad (Cohen, 1988; Gobi, 2003; Kim, Chang y Huh, 2011; McCannell, 1973; Wang, 1999). En este sentido, esa búsqueda no sería propia de los couchsurfers, quienes no se distinguirían de los turistas en general.

Lo que los couchsurfers parecen estar señalando como propio y distintivo de ellos es el intercambio sociocultural, lo que parece distinguirlos del turista convencional. Sin embargo, existen distintos tipos de turismo donde el intercambio es un eje importante, tal como el turismo sustentable y comunitario (cfr. por ejemplo Asociación de Estados del Caribe, 2012 y Moragues Cortada, 2006) y el turismo de raíces o diásporas (cfr. por ejemplo Cardoso, Matos Pereira y Marques, 2018 y Perelló Cabrera, 2015).

El análisis de los cuestionarios online complementó las respuestas de los entrevistados sobre la búsqueda de la autenticidad, viendo que el 57,14\% de la muestra sostuvo que su motivación a participar de Couchsurfing radicaba en la idea 
Innovación y Desarrollo Tecnológico y Social (2020) 2 (1): 1-34

de tener experiencias locales auténticas y el $68,57 \%$ sostuvo que lo motivaba conocer personas y otras culturas (Tabla 1).

\section{Tabla 1: Motivación de los couchsurfers}

\begin{tabular}{|c|c|c|}
\hline Motivación de los couchsurfers & Rango general $(n=70)$ & $\begin{array}{l}\% \text { de la } \\
\text { muestra }\end{array}$ \\
\hline $\begin{array}{l}\text { Quería conocer gente y otras } \\
\text { culturas }\end{array}$ & 48 & 68,57 \\
\hline $\begin{array}{l}\text { Quería tener experiencias locales y } \\
\text { auténticas }\end{array}$ & 40 & 57,14 \\
\hline $\begin{array}{l}\text { Iba a emprender un viaje y no tenía } \\
\text { mucho presupuesto }\end{array}$ & 33 & 47,14 \\
\hline $\begin{array}{l}\text { Quería recibir gente de otros países } \\
\text { en mi casa }\end{array}$ & 23 & 32,86 \\
\hline Quería hacer amigos & 9 & 12,86 \\
\hline $\begin{array}{l}\text { Estaba buscando alojamiento } \\
\text { barato }\end{array}$ & 8 & 11,43 \\
\hline $\begin{array}{l}\text { Me pareció que era una plataforma } \\
\text { responsable con el medio ambiente } \\
\text { y las comunidades locales }\end{array}$ & 5 & 7,14 \\
\hline Me invitaron & 1 & 1.43 \\
\hline $\begin{array}{l}\text { Soy docente de inglés y quería } \\
\text { perfeccionarlo practicando }\end{array}$ & 1 & 1,43 \\
\hline Me dio curiosidad cómo funcionaba & 1 & 1,43 \\
\hline $\begin{array}{l}\text { Estaba viajando solo y en mi } \\
\text { primera ciudad no conocí a nadie, } \\
\text { así que busqué esta opción }\end{array}$ & 1 & 1,43 \\
\hline
\end{tabular}

Asimismo, cuando se les preguntó cómo definirían Couchsurfing, el 91,4\% de los encuestados sostuvo que era una plataforma que promovía el intercambio cultural y el $85,7 \%$ dijo que era un medio para conocer personas de otras culturas. Esto también se mostró en las expresiones que utilizaron los encuestados para describir sus experiencias, siendo las más repetidas compartir, intercambio, enriquecedora, 
Innovación y Desarrollo Tecnológico y Social (2020) 2 (1): 1-34

aprendizaje, amistad, hospitalidad, cultural, local, aventura y solidaridad, entre otras relacionadas.

\section{Couchsurfing como innovación}

Teniendo en cuenta la concepción de innovación del Manual de Oslo con orientación al mercado, podríamos decir que el modelo de Couchsurfing no se encuadra dentro de esta definición, en principio porque nació como una organización sin fines de lucro que buscaba satisfacer una demanda específica, pero no generaba ingresos para sus creadores. Por el contrario, representaba pérdidas en lo que respecta al costo de mantenimiento que se necesitaba para mantener la plataforma en funcionamiento.

Sin embargo, hay ciertas características de las innovaciones que podemos observar en Couchsurfing, por ejemplo, que la creación de la plataforma ha requerido de conocimientos técnicos para su funcionamiento y que ha tenido resultados favorables, ya que incrementó considerablemente el número de participantes en pocos años. Por otro lado, si consideramos los aspectos sociales de la innovación, Couchsurfing, de acuerdo con lo que los usuarios señalan, responde a una necesidad que no es satisfecha desde otros espacios y en ese sentido constituye una innovación.

Si tomamos la conceptualización de Echeverría (2008), se puede sugerir que este modelo Couchsurfing es una innovación social. Desde sus comienzos nace como una comunidad global que promueve ciertos valores sociales y culturales a través de los intercambios de alojamiento y hospitalidad y actualmente cuenta con catorce millones de usuarios, lo que indica que existe un enorme grupo de personas con necesidades asociadas a este espacio y que al participar en esta comunidad se beneficia. Se podría sugerir que genera bienestar social y, en ese sentido, al ser una tecnología que da respuesta a una necesidad no cubierta por otros espacios sociales y económicos, podría ser una innovación tecnológica social. Además, a razón de que 
uno de sus principales propósitos es el intercambio cultural a través del contacto con personas de distintos puntos geográficos y de compartir el hogar, también podría ser pensada como una innovación cultural.

Podemos pensar a Couchsurfing como innovación tecnológica, social y cultural. Tecnológica porque la creación de la plataforma virtual constituye un cambio a partir de conocimientos técnicos y tecnológicos, social porque genera bienestar a partir de satisfacer la necesidad de un grupo de personas en particular y cultural porque promueve el intercambio entre pobladores de distintos espacios geográficos y da lugar a tener la oportunidad de experimentar situaciones locales y auténticas, según los couchsurfers.

Como innovación, el modelo que propone Couchsurfing también podría pensarse como un modo de turismo, aunque no es el modo de ser concebido por los usuarios. Esto puede responder a la conceptualización de turismo que los participantes de couchsurfing tienen.

Según lo analizado, el turismo para los couchsurfers responde a una concepción tradicional, el turismo vinculado con conocer sitios históricos o culturales desde una perspectiva externa, el turismo de masas y lo que se puede realizar en un tour turístico tradicional, el turismo asociado a la foto, al recorrer varias capitales europeas en un mismo viaje al Viejo Continente, un turismo de espectáculo, más asociado a las formas de los años '80 del siglo pasado y que sigue existiendo pero que se ha ido desdibujando frente a las nuevas modalidades de turismo. Frente a las formas más tradicionales del turismo, en la década del '90 comienzan a darse nuevas formas de turismo (Rodriguez Darias, Santana Talavera y Diaz Rodriguez, 2010).

El estudio fenomenológico del turismo puede aportar a una nueva mirada donde se ponga el acento en las prácticas de las personas más que en los conceptos, poniendo el énfasis en lo local, los imaginarios sociales, los hechos concretos y 
situados y la comprensión de los fenómenos en el ámbito en el que ocurren. Una perspectiva fenomenológica se desprende de las grandes conceptualizaciones y encasillamientos para situarse en lo cotidiano y los fenómenos culturales situados que acercan al turismo a la sustentabilidad social (ejemplos de estudios fenomenológicos en turismo pueden encontrarse en Castro y Luna Molina, 2018 y Molina, 2019).

Es más, los cambios tecnológicos y socioculturales han dado lugar a hablar de un pos-turismo (Bezerra, Melo Silva Luft y Rocha Dacoroso, 2012). El pos-turismo se presenta como un nuevo paradigma impulsado por las innovaciones tecnológicas de los últimos tiempos, que surge a finales de la década de los '90 como una forma de entender la influencia de las tecnologías de la información y la comunicación en las innovaciones del sector turístico. Este paradigma comprende el conjunto de cambios adoptados por el turismo frente a la dinámica de los escenarios sociales, organizacionales y culturales relacionada con el paso del tiempo y las nuevas tecnologías.

\section{Conclusiones}

Si lo que define un comportamiento es lo que las personas entienden por el mismo, Couchsurfing podría no ser entendido como turismo. Sin embargo, esto depende de qué mirada tengamos sobre el turismo como actividad humana.

Desde una aproximación no lineal sino compleja del turismo (cfr. por ejemplo Jere Jakulin, 2016) puede sostenerse que esta actividad constituye un subsistema social en interrelación compleja con otros subsistemas, de manera que un cambio, aunque menor en un subsistema asociado afectará al turismo de una forma no fácilmente predecible. El turismo es parte del mundo real que cambia al alterar sus relaciones entre sus subsistemas, así como sus interacciones con el medioambiente. 
Innovación y Desarrollo Tecnológico y Social (2020) 2 (1): 1-34

Los cambios tecnológicos y sociales han afectado la forma de las personas de comportarse frente a sus necesidades y deseos y las formas de satisfacer sus inquietudes. El turismo puede considerarse, entonces, un ámbito más creado por las personas y por ende en movimiento de acuerdo a los cambios que en distintos ámbitos las personas generan. Esto no es ajeno a los modelos de turismo que utilizan las tecnologías de lo información y comunicación como Couchsurfing y pueden, desde esta perspectiva, considerarse una forma alternativa de turismo definido de forma sistémica y compleja. Dentro del paradigma del pos-turismo, las modalidades de turismo nuevas y mediadas por las tecnologías informáticas, como Couchsurfing, representan innovaciones surgidas frente a necesidades de usuarios dentro de contextos cambiantes y dinámicos y pueden considerarse formas originales de realizar una actividad humana como el turismo que, con el tiempo, también ha adquirido nuevos modos de conceptualizarse.

\section{Limitaciones de la investigación}

Los resultados de este trabajo no son generalizables a la comunidad de couchsurfers. Son necesarias más investigaciones con distintos métodos para arribar a resultados más potentes.

\section{Conflictos de interés}

Las autoras no declaran conflictos de interés, no están asociadas a ninguna empresa de turismo ni han recibido ningún tipo de apoyo económico ni soporte de otro tipo de Couchsurfing para la realización de este trabajo.

\section{Información sobre el patentamiento/registro de la innovación o desarrollo}

El presente trabajo no se refiere a una innovación propia y el patentamiento / copyright no es injerencia de las autoras. Couchsurfing tiene su propio 
patentamiento (Couchsurfing Copyright Agent, CT Corporation Systems, LoS Angeles, CA 90017, cfr. http://www.couchsurfing.com/about/terms-of-use/).

\section{Referencias bibliográficas}

Álvarez Sousa, A., Rego Veiga, G., Leira López, J., Gomis Rodríguez, A., Caramés Valo, R. y Andrade Suárez M. J. (2008). Innovación turística: perspectivas teóricas y objetos de estudio. ROTUR. Revista de ocio y turismo, 1(1), 19-50. Recuperado desde: http://revistas.udc.es/index.php/rotur/article/view/1224

Asociación de Estados del Caribe. (2012). Turismo comunitario. Recuperado desde: http://www.acs-aec.org/index.php?q=es/sustainable-tourism/el-turismocomunitario

Ascanio, A. (2007). Reseña de "Proyecto CouchSurfing. Una red de intercambio de viajeros". PASOS. Revista de Turismo y Patrimonio Cultural, 5(3), 407-408. Recuperado desde: http://www.scielo.org.ar/pdf/eypt/v26n2/v26n2a06\%20.pdf

Bezerra, E. D., Melo Silva Luft, M. C. y Rocha Dacoroso, A. L. (2012). El turismo en la sociedad de la información. Un abordaje conceptual sobre el "pos-turismo". Estudios $y$ Perspectivas en Turismo, 21, 1262-1280. Recuperado desde: http://estudiosenturismo.com.ar/PDF/V21/N05/v21n5a11.pdf

Castro, H. R. y Luna Molina, E. O. (2018). La perspectiva fenomenológica como aporte al desarrollo sostenible del turismo. CONDET: Realidad, Tendencias y Desafíos en Turismo, 16(1), 128-143. Recuperado desde: http://revele.uncoma.edu.ar/htdoc/revele/index.php/condet/article/view/2007/584 $\underline{53}$

Cohen, E. (1988). Authenticity and commoditization in tourism. Annals of Tourism Research, 15, 371-386. doi: 10.1016/0160-7383(88)90028-X 
Innovación y Desarrollo Tecnológico y Social (2020) 2 (1): 1-34

Couchsurfing (2019). Página oficial de Couchsurfing. http://www.couchsurfing.com/about/

Drumond, A. (2013). Contextualizing Authenticity in Tourism: An Examination of Postmodern Tourism Theory. CCTP725: Cultural Hybridity: Remix and Dialogic Culture. Georgetown University. Recuperado desde:

https://blogs.commons.georgetown.edu/cctp-725-

fall2013/2013/12/14/contextualizing-authenticity-in-tourism-an-examination-ofpostmodern-tourism-theory/

Dujmović, M. y Vitasović, A. (2015). Postmodern Society and Tourism. Journal of Tourism and Hospitality Management, 3(9-10), 192-203. doi: 10.17265/2328$2169 / 2015.10 .003$

Echevarría, J. (2008). El manual de Oslo y la innovación social. Arbor, 184(732), 609618. Recuperado desde:

http://arbor.revistas.csic.es/index.php/arbor/article/view/210

Escobar Yéndez, N. V. (2000). La innovación tecnológica. Medisan, 4(4), 3-4. Recuperado desde: http://bvs.sld.cu/revistas/san/vol4 4 00/san01400.htm

Gibson, R. (junio de 2014). Rowan Gibson: "Una idea se convierte en innovación cuando crea valor". Colombia: Grandes Realidades, Argos. Recuperado desde: http://grandesrealidades.argos.co/rowan-gibson-una-idea-se-convierte-eninnovacion-cuando-crea-valor/

Gobbi, J. (octubre, 2003). Turismo y autenticidad: hacia una propuesta relacional para el estudio de la interacción entre nativos y turistas en las comunidades locales. Trabajo presentado en el III Encuentro de Turismo Cultural-NayA, Buenos Aires. Recuperado desde:

http://www.equiponaya.com.ar/turismo cultural/congreso2003/ponencias/Jorge G obbi.htm 
Innovación y Desarrollo Tecnológico y Social (2020) 2 (1): 1-34

Fuller, N. (2015). El debate sobre la autenticidad en la antropología del turismo. Revista de Antropología Experimental, (15), 101-108. doi: 10.17561/rae.v0i15.2378

Gómez y Patiño, M., Medina, F. y Puyuelo Arilla, J. (2016). New trends in tourism? From globalization to postmodernism. International Journal of Scientific Managment Tourism, 2016, 2(3), 417-433. Recuperado desde: http://www.ijosmt.com/index.php/ijosmt/article/view/147/154

Hiernaux-Nicolás, D. (2002). ¿Cómo definir el turismo? Un repaso disciplinario. Aportes y Transferencias, 6(2), 11-27. Recuperado desde: http://nulan.mdp.edu.ar/258/1/Apo2002a6v2pp11-27.pdf

Ibáñez, R., y Rodríguez, I. (2012). Tipologías y antecedentes de la actividad turística: turismo tradicional y turismo alternativo. Medio ambiente y política turística en México, 1, 17-33. Recuperado desde: https://micrositios.inecc.gob.mx/publicaciones/libros/669/tipologias.pdf Jere Jakulin, T. (2016). Systems approach for contemporary complex tourism systems. International Journal for Quality Research, 10(3), 511-522. doi: 10.18421/IJQR10.03-05

Jiménez, M. T. (septiembre de 2015). Creatividad + Valor Añadido = Innovación. España: El Mundo. $\quad$ Recuperado desde: https://www.elmundo.es/economia/2015/09/18/55fbde43ca4741af388b457f.html

Kim, H., Chang, H. y Huh, C. (2011). The Relationship between Types of Tourist and Destination Authenticity. University of Massachusetts Amherst. Recuperado desde: https://scholarworks.umass.edu/gradconf hospitality/2011/Poster/72/

MacCannell, D. (1973). Staged Authenticity: Arrangements of Social Space in Tourist Settings. American Journal of Sociology, 79(3), 589-603. Recuperado desde: http://www.jstor.org/stable/2776259 
Innovación y Desarrollo Tecnológico y Social (2020) 2 (1): 1-34

Maguire, M. y Delahunt, B. (2017). Doing a thematic analysis: A practical, step by step guide for learning and teaching scholars. AISHE-J, 8(3), 1-14. Recuperado desde: http://ojs.aishe.org/index.php/aishe-j/article/view/335

Milano, C., Novelli, M. y Cheer, J. M. (2019). Overtourism and Tourismphobia: A Journey Through Four Decades of Tourism Development, Planning and Local Concerns. Tourism Planning \& Development, 16(4), 353-357. doi: 10.1080/21568316.2019.1599604

Molina, M. E. (2019). Un acercamiento teórico a la significación del turismo desde lo fenomenológico. PASOS: Revista de Turismo y Patrimonio Cultural, 17(1), 9-23. doi: 10.25145/j.pasos.2019.17.001

Moragues Cortada, D. (2006). Turismo, cultura y desarrollo. OEI. Recuperado desde: https://www.oei.es/historico/cultura/turismodmoragues.htm

National Commission for the Protection of Human Subjects of Biomedical and Behavioral Research. (1979). The Belmont report: Ethical principles and guidelines for the protection of human subjects of research. Recuperado desde: https://www.hhs.gov/ohrp/regulations-and-policy/belmont-report/read-thebelmont-report/index.html

OCDE (1996). La innovación tecnológica: definiciones y elementos de base. Redes, 3 (6), 131-175. Recuperado desde:

http://www.redalyc.org/articulo.oa?id=90711287005

OCDE y Comunidades Europeas (2006). Manual de Oslo: Guía para la recogida e interpretación de datos sobre innovación, 3era edición. París-Bruselas: OCDEEUROSTAT. Recuperado desde: http://ctie.economia.cl/2017/07/26/manual-de-oslo/ Perelló Cabrera, J. L. (2015). Contribución de la diáspora cubana al crecimiento del turismo internacional en Cuba. Revista de Investigación en Turismo, 5(2), 21-31. Recuperado desde: http://revistes.ub.edu/index.php/ara/article/view/19062 
Innovación y Desarrollo Tecnológico y Social (2020) 2 (1): 1-34

Pérez-García, A. y García Abad, L. (2018). Turismofobia: presencia, impacto y percepción del concepto a través de los medios de comunicación impresos. adComunica. Revista Científica de Estrategias, Tendencias e Innovación en Comunicación, 16, 201-219. doi: 10.6035/2174-0992.2018.16.11

Rodriguez Darias, A. J., Santana Talavera, A. y Diaz Rodriguez, P. (2010). Las nuevas formas de turismo: causas y características. Revista Brasileira de Pesquisa em Turismo, 4(3), 54-70. $\quad$ Recuperado desde: https://pdfs.semanticscholar.org/91b5/1e18cb781760d971c405eee717563428af11 . $\mathrm{pdf}$

Ryan, G. W. y Bernard, H. R. (2003). Techniques to identify themes. Field Methods, 15(1), 85-109. doi: 10.1177/1525822X02239569

Spencer-Oatey, H. (2012). What is culture? A compilation of quotations. GlobalPAD Core Concepts. GlobalPAD Open House. Recuperado desde: https://warwick.ac.uk/fac/soc/al/globalpad/openhouse/interculturalskills/global pa d - what is culture.pdf

Terziysca, I. (2012). Interpretations of authenticity in tourism. Science\&Research, 4, 85-94. Recuperado desde: https://www.researchgate.net/publication/280304792 INTERPRETATIONS OF AUT HENTICITY IN TOURISM

Wang, N. (1999). Rethinking Authenticity in Tourism Experience. Annals of Tourism Research, 26(2), 349-370. doi: 10.1016/S0160-7383(98)00103-0 